\section{Ulcer therapy gets thumbs up}

Forest Laboratories says its mouth ulcer treatment Aloclair has been accredited by the Dental Health Foundation.

The liquid, which provides fast pain relief, is the only product of its kind to be approved by the foundation, said the firm. Aloclair contains a barrier forming agent that puts a coating over the exposed ulcer and the nerve endings underneath. A 60-second rinse with Aloclair reduces the irritation and discomfort of mouth ulcers, said the firm. One in five adults suffers from mouth ulcers in the UK. Dispensers with free $10 \mathrm{ml}$ sachets are available from Forest Laboratories UK on 01322550550.

\section{Keep your edge with sharper tools}

Every dentist knows that sharp instruments are vital for the efficient removal of deposits from tooth and root surfaces.

To keep instruments in perfect condition, Dentsply says tools should be stored on a rack to reduce the risk of contamination and damage. Instruments or tips should be replaced when worn or damaged and blunt instruments must be sharpened.

Dentsply offers a sharpening service for its Ash range of instruments, which aims to return your tools to almost new. If you would rather sharpen your instruments in the surgery, Dentsply will send a specialist to demonstrate the best techniques. To contact call 01752709751. marke† place?

\section{Give your surgery a designer image}

Do you need to attract new patients to your practice? Marketing and print experts Designer Dental offer eye catching promotional and marketing material tailored to your practice.

The firm provides a

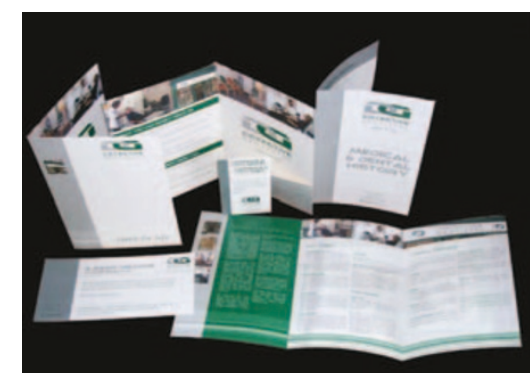
range of marketing services including comprehensive welcome packs for new patients to establish a strong practice-patient relationship. A targeted campaign will educate patients about your practice so they know everything from information on payments to directions to the clinic. To contact call 01642206106.

\section{Injection system}

\section{makes jabs easier}

Administering an anaesthetic using a traditional syringe can cause unnecessary pain. A pain-free injection relaxes the patient and makes treatment easier for the dentist.

DPS offers a computer-controlled Wand local anaesthetic delivery system, which they say makes injections easier and reduces stress for the operator and the patient.

The first step in the process is to apply a topical anaesthetic to freeze the gum. A tiny needle is gently introduced through the already-numb tissue to anaesthetise the surrounding area.

The manufacturers said the injector needs less penetration force. And the controlled flow eliminates the 'bee sting' effect caused by a traditional syringe - which many patients will be pleased to hear. To contact call 01438820550 . 


\section{Create a new look surgery}

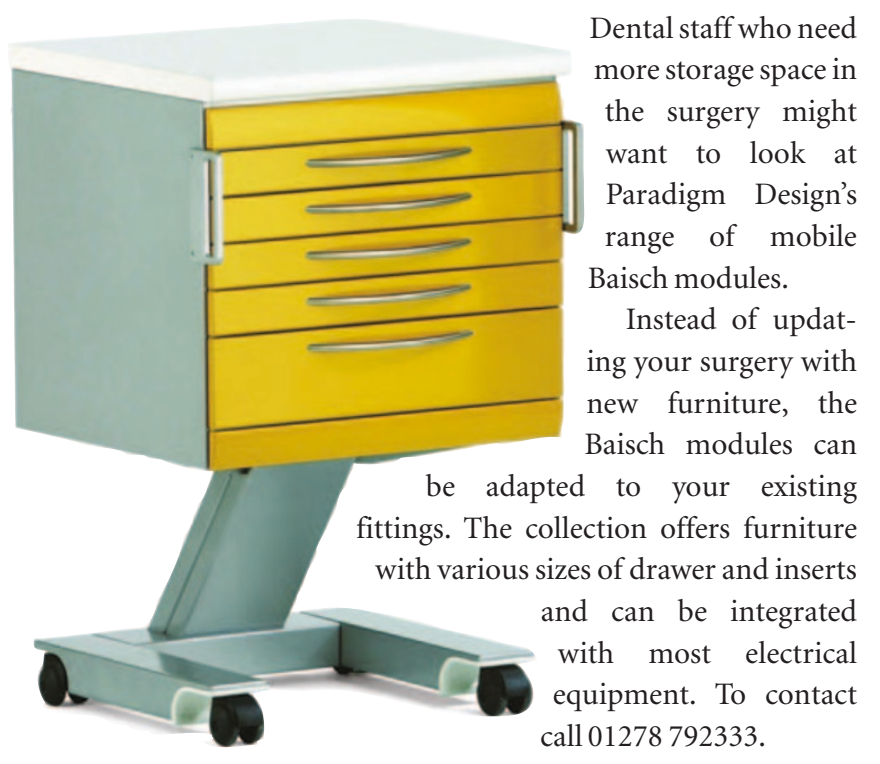

\section{Citrus flavoured}

mouthwash

\section{launched}

Pfizer Consumer

Healthcare has

launched a mild tasting Cool Citrus Listerine, which it hopes will attract new patients to the brand.

The mouthwash contains the same active ingredients as the other flavours in the range - including eucalyptol and menthol - and will reduce plaque when used with regular brushing and flossing. To contact call 020 78153900 .

\section{Powder makes an impression}

Dentsply's Blueprint Cremix is a light, wettable powder, which is lead-free, easy to mix, and suitable for full and partial oral impressions.

Its dust-free consistency minimises eye irritation and powder inhalation during mixing. The dental products company said the powder guarantees high precision impressions and allows ample working time and a short setting time, which reduces the risk of distortion. To contact call 01932837235

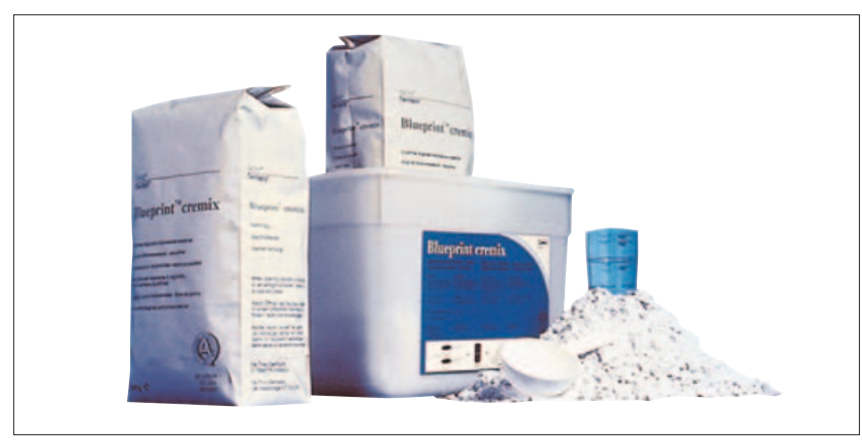

\section{Saddle up on novel seat}

Bambach UK says the innovative design of its Saddle Seat relieves and prevents back pain and is especially good for pregnant dentists.

The extra weight of a baby shifts the centre of gravity forward and puts more strain on the lower back, according to research. $47 \%$ of pregnant dentists get back pain and women who have had back pain during pregnancy are at increased risk

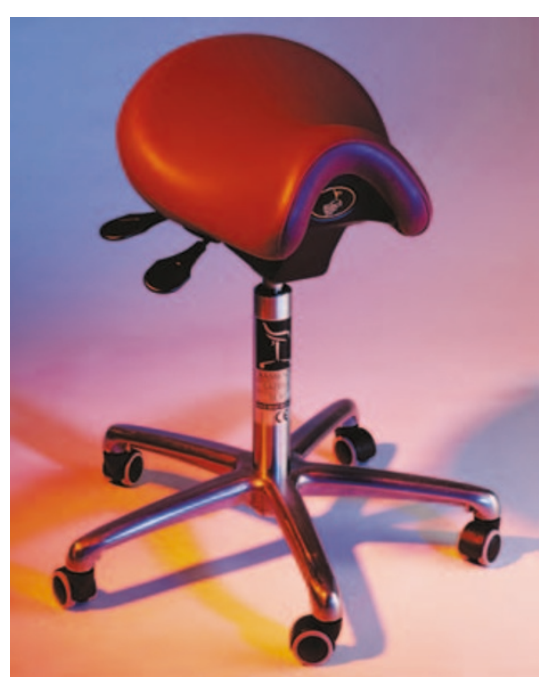
during subsequent pregnancies, they said.

The seat is designed to improve the user's posture by supporting the pelvis and putting the spine in a more natural position. All the chairs in the range allow complete mobility around the dental surgery. To contact call 02085325100. 


\section{Dental Team Books}

\section{Electronic pen simplifies note taking}

Kodak Dental System says its QuickPen tool provides a fast, simple and precise alternative to registering patient records.

Even technophobes should be able to use the QuickPen and its small display. The system can efficiently update patients' charts and calculate fees. It can also be programmed and customised to suit private and NHS treatments, and the accompanying tablet PC style display has a wipe clean surface for better infection control. To contact call 08001699692.

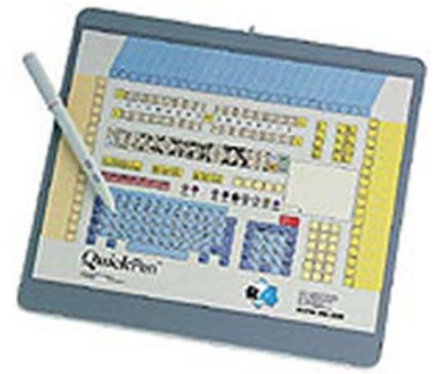

\section{Film packets reduce contamination risk}

Kodak Dental Systems have introduced ClinAsept barrier packets for their range of camera film to minimise the risk of film cross-contamination during intraoral examinations and darkroom processing.

The packets, designed for use with Kodak's Insight's dental films, should help improve hygiene and safety in the dental surgery. The packets guard the films and can be easily removed after exposure and before processing.

Insight Dental Film in ClinAsept packets is available in a $3.1 \times 4.1 \mathrm{~cm}$ size, in boxes of 100 single or double films, and in a $2.2 \times 3.5 \mathrm{~cm}$ size in boxes of 75 single or double films. To contact call 08001699692.

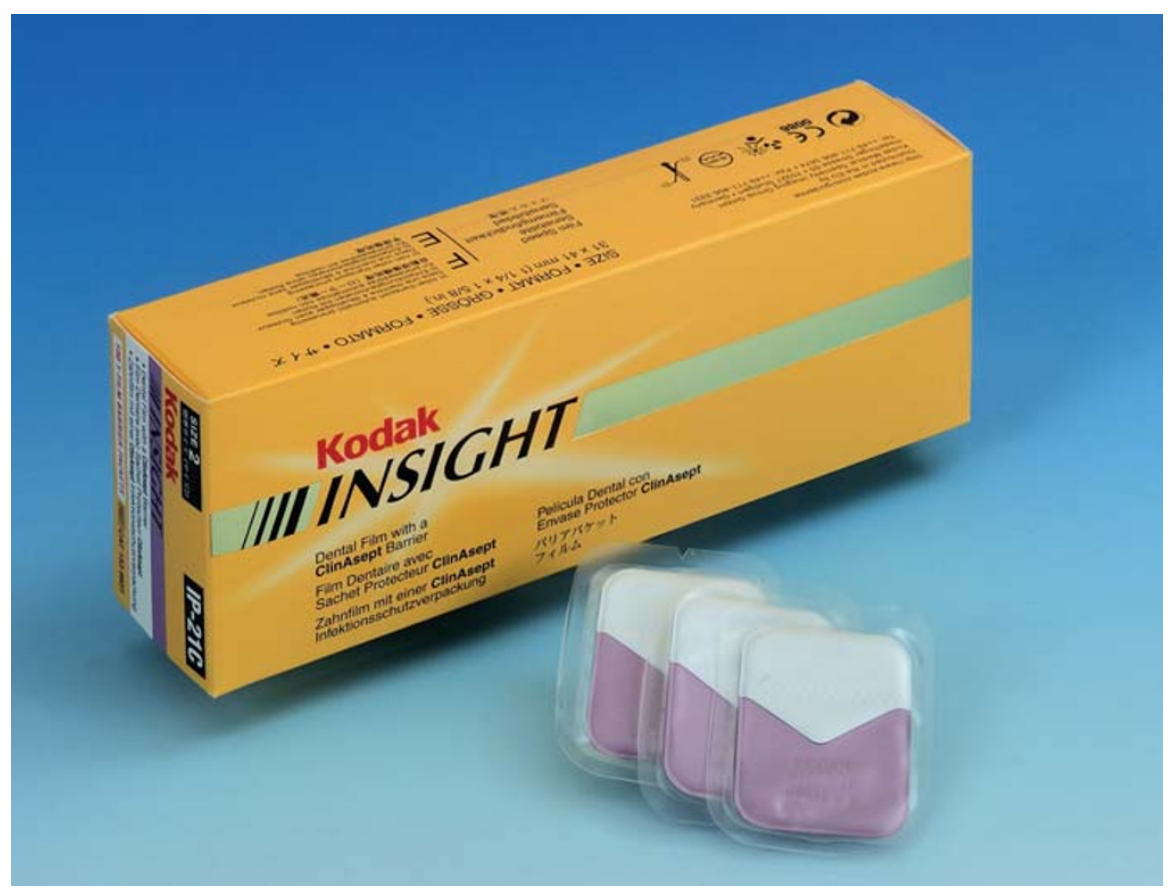

HOW TO ORDER

Dental Team Books

27 Bellamy's Court

Abbotshade Road

London SE16 5RF

Telephone/Fax: +44 (0) 2072322736 